\title{
HIDROFORMILACIÓN EN FASE HOMOGÉNEA DE EUGENOL E ISOEUGENOL CON LOS COMPLEJOS $\left[\mathrm{RhCl}\left(\eta^{4}-1,5-\mathrm{COD}\right)(2-\mathrm{ampy})\right]$ y $\left[\mathrm{RhCl}\left(\mathrm{PPh}_{3}\right)_{2}(2 \text {-ampy })_{2}\right]$
}

\author{
HYDROFORMYLATION IN HOMOGENEOUS PHASE OF EUGENOL AND ISOEUGENOL WITH \\ $\left[\mathrm{RhCl}\left(\eta^{4}-1,5-\mathrm{COD}\right)\left(2\right.\right.$-ampy)] and $\left.\left[\mathrm{RhCl}\left(\mathrm{PPh}_{3}\right)_{2} \text { (2-ampy }\right)_{2}\right]$ Complexes \\ Fernando Cuenú Cabezas ${ }^{1-2}$, María Cristina Duque Hoyos ${ }^{1}$, Alberto Bolaños Rivera ${ }^{2}$ \\ Laboratoria de Química Inorgánica y Catálisis, Programa de Química, Universidad del Quindía, Armenia- Quindio. \\ fercuenu@uniquindio.edu.co,mcduque@uniquindio.edu.co \\ Universidad del Valle, Facultad de Ciencia Exactas y Naturales, Departamento de Química, Cali-Valle, albolan@univalle.edu.co
}

Recibido: Enero 19 de 2011

Aceptado: Noviembre 2 de 2011

Correspondencia: Programa de Química, Universidad del Quindio, Carrera 15 calle 12 norte, Armenia Quindío - Colombia, correo electrónico: fercuenu@uniquindio.edu.co

\section{RESUMEN}

Se realizó la síntesis de los complejos $\left[R h C l\left(\eta^{4}-1,5-C O D\right)(2-a m p y)\right]$ y $\left[R h C l\left(P P h_{3}\right)_{2}(2-a m p y)_{2}\right]$, (donde COD es ciclooctadieno, 2-ampy es 2-aminopiridina y $\mathrm{PPh}_{3}$ trifenilfosfina). El compuesto $\left[R \mathrm{RhCl}\left(\mathrm{PPh} \mathrm{C}_{2} \mathrm{I}_{2}(2 \text {-ampy })_{2}\right] \mathrm{presenta}\right.$ un doblete a $29.4 \mathrm{ppm}$, con una constante de acoplamiento $\mathrm{Rh}-\mathrm{P}$ de $86 \mathrm{~Hz}$, indicando que las fosfinas se encuentran en posición trans. Los compuestos fueron activos en la hidroformilación de eugenol e isoeugenol, en donde la selectividad y actividad catalitica depende de varios factores tales como, los impedimentos estéricos en el ligante, los factores electrónicos y la temperatura de reacción. El complejo con los ligantes fosfina presento una menor actividad catalitica pero una mayor selectividad hacia aldehídos lineales.

Palabras clave: Hidroformilación; Eugenol; Isoeugenol; 2-aminopiridina ligando; Rodio; Trifenilfosfina ligando.

\begin{abstract}
In this study, a synthesis with $\left[R h C l\left(\eta^{4}-1,5-C O D\right)\right]$ and $\left[R h C l\left(P P h_{3}\right)_{2}(2-a m p y)_{2}\right](C O D=c y c l o o c t a d i e n e, 2-a m p y=$ 2-aminopyridine and $P P_{3}=$ triphenylphosphine) complexes was conducted. The compound $\left[R h C l\left(P P h_{3}\right)_{2}(2-\right.$ ampy $)_{2}$, presents a doublet to $29.4 \mathrm{ppm}$, with a coupling constant of $\mathrm{Rh}-\mathrm{P}$ of $86 \mathrm{~Hz}$, indicating that the triphenylphosphine are in position trans. Compounds were active in the hydroformylation with eugenol and isoeugenol, in which the selectivity and catalytic activity depend on several factors such as the steric hindrance in the ligand, electronic factors and reaction temperature. The complex with phosphine ligands presented a minor catalytic activity, but a major selectivity towards linear aldehydes
\end{abstract}

Keywords: Hydroformylation, Rhodium, triphenylphosphine ligands, 2-aminopyridine ligands, Eugenol, Isoeugenol.

\section{INTRODUCCIÓN}

Los complejos de metales de transición con ligantes $\mathrm{N}$-heterocíclicos aromáticos tales como piridinas y compuestos relacionados, han demostrado poseer altas actividades catalíticas en diferentes reacciones [1]. Además, estos complejos metálicos con ligantes $\mathrm{N}$-heterocíclicos, poseen una mayor estabilidad que los complejos fosfinados, cuando se trabaja a altas temperaturas, esto es debido a que el átomo de nitrógeno no posee orbitales $d \pi$ disponibles, así, ligantes $\mathrm{N}$ donores se comportan como buenos donores $\sigma$ de densidad electrónica y débiles aceptores $\pi$, lo que genera que el enlace $M-N$, sea de un carácter más iónico que el enlace M-P [2], así a altas temperaturas no hay descomposición del sistema catalítico.

La hidroformilación de alilbencenos, representa un poderoso instrumento sintético para la síntesis de un 
gran número de compuestos fármacologícamente importantes, en este contexto, los aldehídos derivados de los alilbencenos muestran actividades biológicas y fitosanitarias, siendo también útiles en la industria de sabores, perfumes y farmacéutica [3]. A pesar de esto, la hidroformilación de estas olefinas ha sido muy poco estudiada [4-8].

Específicamente, el eugenol, quien es un alilbenceno, es el componente principal de varios aceites esenciales, como el del clavo de olor, y se emplea en perfumería y en odontología como antiséptico. El eugenol $e$ isoeugenol son utilizados como saborizantes para productos como bebidas, dulces y productos refrigerados y son ampliamente usados en cosmética, odontología y medicina tradicional [9].

La literatura reporta muchos trabajos en la hidroformilación de vinil-aromáticos [10], pero pocos trabajos han sido realizados con alilbencenos [11], esto es sorprendente, toda vez que, por ejemplo, la hidroformilación de propenilcencenos, puede generar la formación de una especie opticamente activa, como el 2-fenilbutanal, el cual puede ser oxidado fácilmente al ácido 2-fenilbutanoico, precursor directo del antiinflamatorio no esteroidal, indobufeno [12].

Por lo tanto, en los últimos años se ha intensificado la síntesis de complejos metálicos con ligantes heterocíclicos, para ser utilizados en diferentes reacciones catalíticas $[13,14]$. En este contexto estamos trabajando en la síntesis de complejos de metales de transición con ligantes $\mathrm{N}$-heterocíclicos $\mathrm{y}$ libres de fosfinas, para ser utilizados en reacciones de hidroformilación de alil y propenilbencenos.

\section{PARTE EXPERIMENTAL}

Los solventes y reactivos $[\mathrm{RhCl}(\mathrm{COD})]_{2}, \mathrm{RhCl}\left(\mathrm{PPh}_{3}\right)_{3}, 4$ terbutilpiridina, benceno, tolueno, THF, eugenol, isoeugenol, diclorometano se compraron de las compañías Aldrich Chemical Co, Sigma and Fluka, y se utilizaron como se recibieron.

Benceno se purificó bajo reflujo con sodio metálico y benzofenona por 6 horas y después se destilo bajo una atmósfera de nitrógeno. Diclorometano fue destilado bajo una atmósfera de nitrógeno sobre pentóxido de fósforo. La síntesis de los complejos fue realizada bajo una atmósfera de nitrógeno, empleando técnicas estándar de Schlenk.

Los espectros de NMR fueron obtenidos usando un espectrofotómetro Bruker avance II de $400 \mathrm{MHz}$ en DMSO $d_{6}$ y $\mathrm{CDCl}_{3}$. Para los estudios IR, se empleó un espectrofotómetro infrarrojo con transformada de Fourier marca Shimadzu 3800 con un rango de barrido entre 4000 y $400 \mathrm{~cm}^{-1}$, mediante la técnica de pastillas de $\mathrm{KBr}$. La cromatografía de gases se realizó en un Hewlett Packard 6890 con un detector de ionización de llama; las condiciones de inyección son: temperatura del inyector $300{ }^{\circ} \mathrm{C}$, detector FID a 300 ${ }^{\circ} \mathrm{C}$ con una rampa de temperatura de $50{ }^{\circ} \mathrm{C}$ (1 min), $230^{\circ} \mathrm{C}(2 \mathrm{~min})$ y $300^{\circ} \mathrm{C}(20 \mathrm{~min})$. El gas portador fue helio empleando una presión de 12.1 Psi, con un flujo de $2.0 \mathrm{~mL} / \mathrm{min}$ y una columna HP-5 de $30 \mathrm{~m}$ fase polimetilsilano al $5 \%$. Los masas se obtuvieron en un espectrofotómetro Shimadzu GCMS-QP 2010 operando a $70 \mathrm{eV}$.

Las reacciones de hidroformilación se realizaron en un reactor Parr de $125 \mathrm{cc}$, y las condiciones de reacción son Temperatura $80 \stackrel{\circ}{\circ}$, relación catalizador sustrato 1:3000, solvente benceno $20 \mathrm{~mL}$., tiempo de reacción 5 horas, presión 40 bar $\left(\mathrm{H}_{2} / \mathrm{CO}, 1: 1\right)$.

\section{Síntesis de cloro $\left(\eta^{4}-1,5\right.$-ciclooctadieno) (2-aminopiridina)rodio(I), [RhCl $\left.\left(\eta^{4}-1,5-\mathrm{COD}\right)(2-\mathrm{ampy})\right]$.}

Se pesan 0,1015 g (0,206 mmoles) de $[\mathrm{RhCl}(\mathrm{COD})]_{2} \mathrm{y}$ se adiciona a un balón de $50 \mathrm{~mL}$, el cual contiene 4 $\mathrm{mL}$ de diclorometano previamente desgasificado con Nitrógeno gaseoso, la solución se deja en agitación magnética por dos minutos luego se adiciona 0,0193 g $(0,206$ mmoles) de 2-aminopiridina. La reacción se mantiene en agitación hasta la solución pasa de un color amarillo claro a un café amarillento, tiempo en el cual se ha consumido la 2-ampy, el seguimiento se realiza por cromatografía de capa delgada (10 minutos), la solución se rotavapora a presión hasta sequedad, con la aparición de unos sólidos café claros, los sólidos se lavan éter etílico y hexano y se secan al vacío. El rendimiento de la reacción es del 94\%. Análisis elemental calculado \%C 45.88, \% H 5.29, \% N 8.23; experimental \%C 45.85, \% H 5.32, \% N 8.08; para la fromula molecular $\mathrm{RhClC}_{13} \mathrm{H}_{18} \mathrm{~N}_{2}$. 


\section{Síntesis de cloro \\ (2-aminopiridina)bis(trifenilfosfina)rodio(I), $\left[\mathrm{RhCl}\left(\mathrm{PPh}_{3}\right)_{2}\right.$ (2-ampy)]}

Método 1. Se pesan $0.1012 \mathrm{~g}$ (0.109 mmoles) de $\mathrm{RhCl}\left(\mathrm{PPh}_{3}\right)_{3}$ y se adicionan a un balón de $50 \mathrm{~mL}$ el cual contiene acetona previamente desgasificada con Nitrógeno gaseoso, la solución se deja en agitación magnética por dos minutos luego se adiciona $0.042 \mathrm{~g}$ (0.438 mmoles) de 2-ampy. La reacción se mantiene en agitación hasta la solución pasa de un color rojo a amarillo y con la completa desaparición de la 2-ampy (30 minutos, seguimiento con cromatografía de placa delgada), los sólidos amarillos se filtran al vacío y se lavan con acetona, el rendimiento de la reacción es del $85 \%$.

Método 2. Se pesaron $0.3475 \mathrm{~g}$ (1.026 mmoles) de [RhCl(COD)(2-ampy) ], se adiciona a $3 \mathrm{~mL}$ de diclorometano previamente desgasificado con nitrógeno gaseoso, posteriormente se adiciona $0.5380 \mathrm{~g}$ (3.08 mmoles) de $\mathrm{PPh}_{3}$, disuelto en metanol seco y caliente. La solución inicialmente amarilla pasa a un color café-amarillo después de 10 minutos de reacción con la formación de unos sólidos, los cuales se filtran y lavan con éter y metanol. El rendimiento de la reacción es del $93 \%$. Análisis elemental Calculado \%C 65.07; \%H 4.76; \%N 3.72, experimental
\% $65.12 ; \% \mathrm{H} \mathrm{4.61;} \mathrm{\% N} \mathrm{3.68,} \mathrm{Para} \mathrm{la} \mathrm{formula}$ molecular $\mathrm{RhClC}_{40} \mathrm{H}_{36} \mathrm{~N}_{2} \mathrm{P}_{2}$.

\section{RESULTADOSY DISCUSIÓN}

\section{Síntesis de $\left[\mathrm{RhCl}\left(\eta^{4}-1,5-\mathrm{COD}\right)(2 \text {-ampy })_{2}\right]$.}

Ir.

Algunas frecuencias vibracionales de $\left[\mathrm{RhCl}\left(\eta^{4}-1,5\right.\right.$ COD)(2-ampy $)_{2}$ ], se presentan en la tabla 1 . complejo, registradas en pastillas de $\mathrm{KBr}$. En el espectro Ir del complejo se observa una banda intensa a $3412 \mathrm{~cm}^{-1}$, la cual es asignada a la vibración asimétrica de los enlaces $\mathrm{N}-\mathrm{H}$ pertenecientes al ligando 2aminopiridina, banda que en el ligando libre aparece a $3347 \mathrm{~cm}^{-1}$. Este desplazamiento a mayores frecuencias esta indicando que los enlaces $\mathrm{N}-\mathrm{H}$ en el complejo son más cortos que en el ligando libre. Este desplazamiento es consistente el aumento del carácter positivo de los hidrógenos (aumento del carácter ácido de los protones) y con la disminución del carácter nucleofílico del nitrógeno del grupo amino, debido a que esta introduciendo mayor densidad de carga en el anillo de la piridina. La vibración simétrica de los enlaces $\mathrm{N}-\mathrm{H}$ se encuentra a $3309 \mathrm{~cm}^{-1}$, y en el ligando libre a $3307 \mathrm{~cm}^{-1}$.

Tabla 1. Espectro infrarrojo del complejo $\mathrm{RhCl}(\mathrm{COD})(2$-ampy), registrado en pastilla de $\mathrm{KBr}$.

\begin{tabular}{cccc}
\hline Frecuencia $\left(\mathrm{cm}^{-1}\right)$ & asignación & Frecuencia $\left(\mathrm{cm}^{-1}\right)$ & Asignación \\
\hline $3412 \mathrm{mf}$ & $v_{\text {as }} \mathrm{N}-\mathrm{H}$ & $1443 \mathrm{~cm}^{-1}$ & $\omega \mathrm{CH}_{2}, \mathrm{COD}$ \\
$3309 \mathrm{f}$ & $v_{\mathrm{s}} \mathrm{N}-\mathrm{H}$ & 1325 & $\omega \mathrm{CH}_{2}, \mathrm{COD}$ \\
$3083 \mathrm{~d}$ & $v=\mathrm{C}-\mathrm{H}$ py & $1258 \mathrm{~m}$ & $v \mathrm{C}-\mathrm{N}(2-\mathrm{ampy})$ \\
$3003 \mathrm{~d}$ & $v=\mathrm{C}-\mathrm{H}, \mathrm{COD}$ & $867 \mathrm{~m}$ & $\gamma \mathrm{C}-\mathrm{H}(2-\mathrm{ampy})$ \\
$2950,2990 \mathrm{~m}$ & $v_{\text {as }} \mathrm{C}-\mathrm{H}$ de CH & $840 \mathrm{~d}$ & $\gamma \mathrm{C}-\mathrm{H}(2-\mathrm{ampy})$ \\
$2883,2831 \mathrm{~d}$ & $v_{\mathrm{s}} \mathrm{de} \mathrm{CH} \mathrm{CH}_{2}$ & $775 \mathrm{mf}$ & $\omega \mathrm{N}-\mathrm{H}(2-\mathrm{ampy})$ \\
$1665,1622 \mathrm{mf}$ & $v \mathrm{C}=\mathrm{N}, \mathrm{C}=\mathrm{C}$ de py & $691 \mathrm{~d}$ & $\omega=\mathrm{C}-\mathrm{H}, \mathrm{COD}$ \\
$1593 \mathrm{f}$ & $v \mathrm{C}=\mathrm{N}, \delta \mathrm{N}-\mathrm{H}$ & 442 & $v \mathrm{Rh}-\mathrm{Cl}$ \\
& & & \\
1487 y $1428 \mathrm{f}$ & $v \mathrm{C}=\mathrm{C}$ de COD & &
\end{tabular}


A $3083 \mathrm{~cm}^{-1}$ se observa la frecuencia de estiramiento $=\mathrm{C}-\mathrm{H}$ del anillo piridinico, en el ligando libre aparecen a $3073 \mathrm{~cm}^{-1}$, mostrando que los enlaces $\mathrm{C}-\mathrm{H}$, se han debilitado debido a la pérdida de densidad electrónica, porque el nitrógeno de la piridina esta donando sus pares electrones al rodio.

El estiramiento de los enlaces $=\mathrm{C}-\mathrm{H}$ del $\mathrm{COD}$ se presentan a $3005 \mathrm{~cm}^{-1}$, este desplazamiento a menores frecuencias (desde $3010 \mathrm{~cm}^{-1}$ ligando libre) es consistente con la retro-donación de densidad electrónica por parte del metal, lo que genera una disminución en el orden de enlace y por ende un debilitamiento del enlace $\mathrm{C}=\mathrm{C}$, reflejándose en la disminución de la frecuencia de vibración del enlaces $=\mathrm{C}-\mathrm{H}$.

Los estiramientos asimétricos y simétricos de los enlaces $\mathrm{C}-\mathrm{H}$ de los grupos metilénicos se observan a 2990,2950 y $2883,2831 \mathrm{~cm}^{-1}$ respectivamente.

\section{RMN}

\section{RMN ${ }^{1} \mathrm{H}$}

El espectro protónico del complejo presenta siete señales (figura 1), y en la taba 2 se presentan los desplazamientos químicos, el espectro fue registrado en DMSO $d_{6}$.

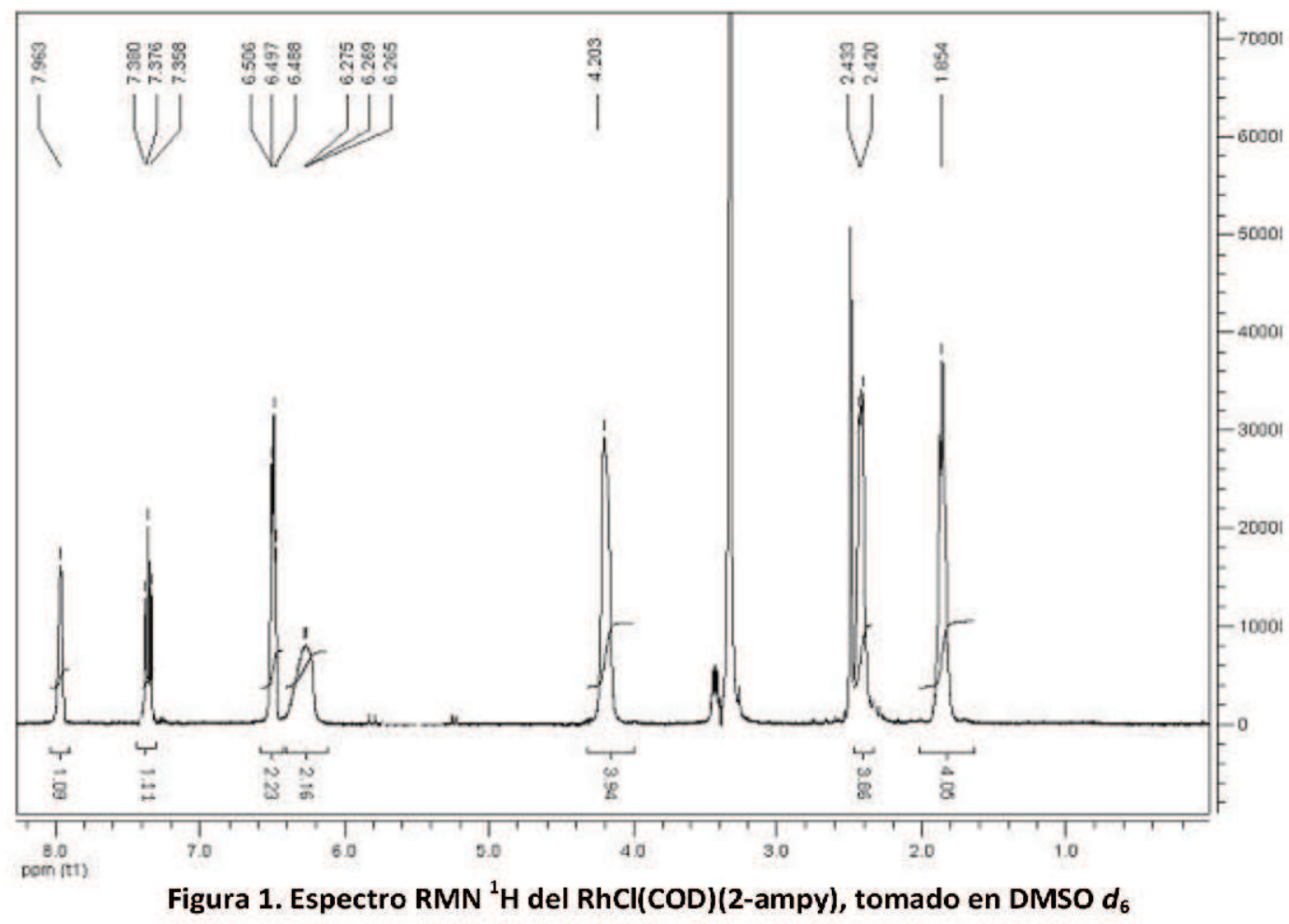

En las figuras 1 y 2 se presenta el espectro de rmn del complejo en la región de 8.30 y 7.90 ppm en donde se observa una señal a 7.96 ppm que integra para un protón, asignado al hidrógeno orto al nitrógeno piridiníco, $\mathrm{Ha}$ del esquema 1, en el ligante libre se observa como un doblete a $8.04 \mathrm{ppm}$. La figura 2 se puede observar que la señal ya no se observa como un doblete. 


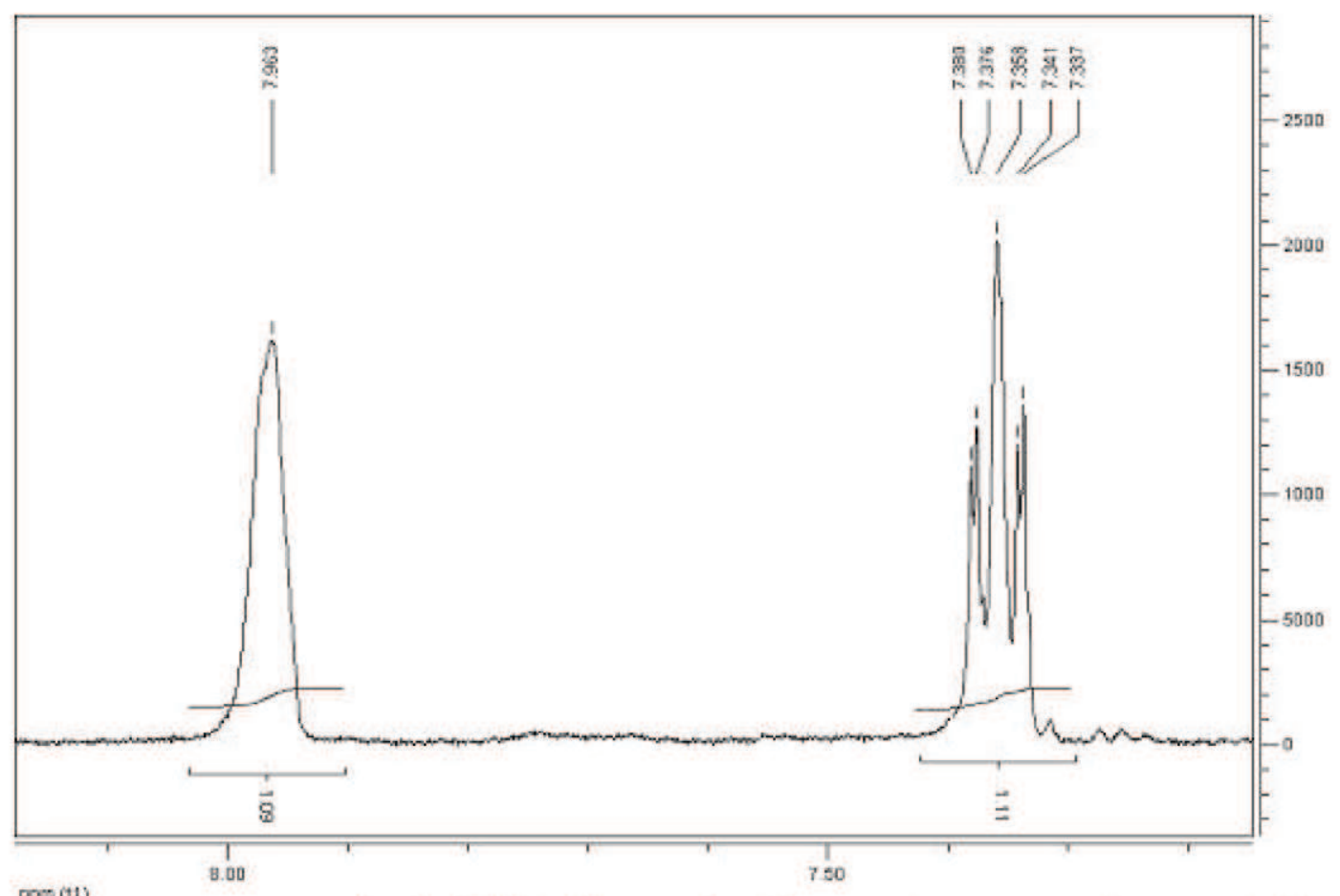

Figura 2. Espectro $\mathrm{RMN}^{1} \mathrm{H}$ de $\mathrm{RhCl}(\mathrm{COD})\left(2\right.$-ampy), región 8.1 a $6.6 \mathrm{ppm}$. tomado en DMSO $d_{6}$

Tabla 2. Espectroscopia $\mathrm{RMN}^{1} \mathrm{H}$ del complejo [RhCl( $\left.\eta^{4}-1,5-\mathrm{COD}\right)(2$-ampy)]

\begin{tabular}{cccc}
\hline ASIGNACIÓN & $\delta, p p m$ & ASIGNACIÓN & $\delta, p p m$ \\
\hline $1 \mathrm{H}$, Ha de 2-ampy & 8.01 & $4 \mathrm{H}, \mathrm{C}-\mathrm{H}, \mathrm{COD}, \mathrm{s}$ & 4.20 \\
$1 \mathrm{H}, \mathrm{Hc}$ de 2-ampy & 7.36 & $4 \mathrm{H}, \mathrm{CH}_{2}, \mathrm{COD}, \mathrm{s}$ & 2.42 \\
$2 \mathrm{H}, \mathrm{Hb}$ y Hd de 2-ampy & 6.49 & $4 \mathrm{H}, \mathrm{CH}_{2} \mathrm{COD}, \mathrm{d}$ & 1.84 \\
$2 \mathrm{H}, \mathrm{NH}_{2}$, de 2-ampy & 6.26 & & \\
\hline
\end{tabular}

$\mathrm{d}=$ doblete, $\mathrm{m}=$ multiplete, $\mathrm{s}=$ singulete.

En la figura 2 se observa además un multiplete que integra para un protón, en 7,36 ppm, siendo asignado a la hidrógeno $\mathrm{Hc}$ de la piridina. en el ligante libre se observa a 7,37 ppm.

Los protones $\mathrm{Hb}$ y $\mathrm{Hd}$, se observan como un multiplete a $6.49 \mathrm{ppm}$ en el ligante libre se observan a 6.67 y $6.46 \mathrm{ppm}$, respectivamente.<smiles>Cc1c(O)c(O)nc(N)c1Cl</smiles>

Esquema 1. Estructura de 2-ampy
Los protones del grupo amino se observan a 6.28 ppm, integrando para dos protones. En el ligante libre se observan a $4.65 \mathrm{ppm}$. Lo anterior es consistente con la pérdida de densidad de carga, generada por la donación de densidad electrónica al anillo de la piridina, por parte del grupo amino, esto confirma lo observado en el espectro infrarrojo, en donde los hidrógenos aumentaban su carácter ácido, es decir su carácter positivo.

\subsubsection{RMN ${ }^{13} \mathrm{C}$}

La figura 3, muestra el espectro de $\mathrm{RMN}{ }^{13} \mathrm{C}$, en donde se observan las mismas señales de los ligantes libre con desplazamientos indicando la coordinación 
al metal, en la tabla 3 se presenta los señal del carbono cuaternario del anillo del ligante 2desplazamientos químicos y las asignaciones de las ampy, $C_{1}$ del esquema 2 , en el ligando libre se señales. Por ejemplo, a $159.67 \mathrm{ppm}$ se observa la encuentran a $158.66 \mathrm{ppm}$.

Tabla 3. Espectro de $\mathrm{RMN}{ }^{13} \mathrm{C}$ del complejo [RhCl( $\left.\eta^{4}-1,5-\mathrm{COD}\right)(2$-ampy)]

\begin{tabular}{cccc}
\hline Señal (en ppm) & asignación & Señal (en ppm) & asignación \\
\hline 159.67 & $\mathrm{C}_{1}$ de la 2-ampy & 109.62 & $\mathrm{C}_{5}$ de 2-ampy \\
147.96 & $\mathrm{C}_{2}$ de 2-ampy & 83.73 & $=\mathrm{CH}$ de COD \\
137.64 & $\mathrm{C}_{3}$ de 2-ampy & 30.77 & $\mathrm{CH}_{2}$, de COD \\
112.67 & $\mathrm{C}_{4}$ de 2-ampy & & \\
\hline
\end{tabular}

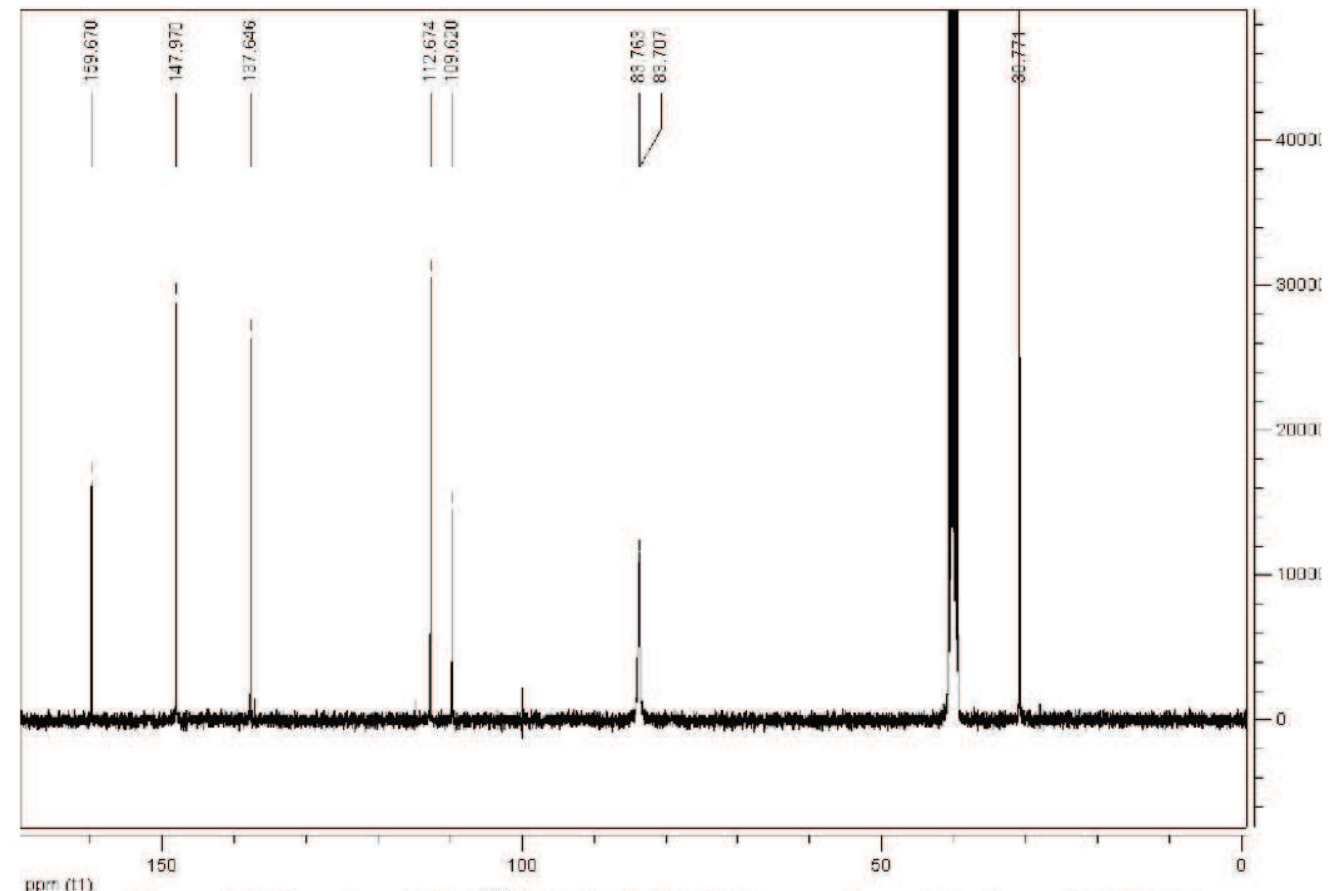

Figura 3. Espectro RMN ${ }^{13} \mathrm{C}$ de RhCl(COD)(2-ampy), registrado en DMSO $d_{6}$

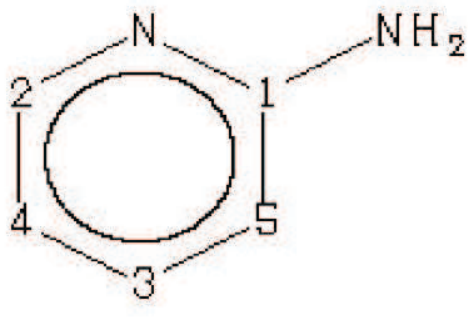

Esquema 2. Estructura de la 2-ampy

Los Datos espectroscópicos son consistentes con una geometría cuadrado planar para el rodio, como se muestra en el esquema 3.

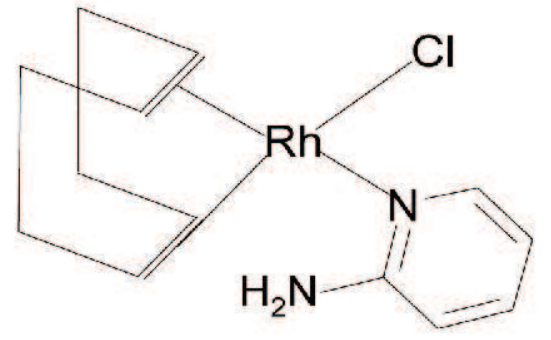

Esquema 3. Estructura del complejo.

cloro $\left(\eta^{4}-1,5\right.$-ciclooctadieno)(2-aminopiridina)rodio(I) $\mathrm{RhCl}\left(\eta^{4}-1,5-\mathrm{COD}\right)(2$-ampy) 


\subsection{Síntesis de $\left[\mathrm{RhCl}\left(\mathrm{PPh}_{3}\right)_{2}(2 \text {-ampy })_{2}\right]$.}

\subsubsection{Ir.}

El espectro Ir del complejo se registro en pastilla de $\mathrm{KBr}$, observándose desplazamientos en las frecuencias vibracionales de los ligandos trifenilfosfina y 2-aminopiridina, mostrando así la coordinación con el metal ver figura 4 y tabla 4 .

En el espectro Ir del complejo se observa una banda intensa a $3395 \mathrm{~cm}^{-1}$ la cual es asignada a la vibración asimétrica del grupo $\mathrm{NH}_{2}$, del ligando 2-ampy, banda que en el ligando libre aparece a $3347 \mathrm{~cm}^{-1}$. De igual manera como ocurre con el complejo $\mathrm{RhCl}(\mathrm{COD})(2$ ampy), el enlace $\mathrm{C}-\mathrm{H}$, se debilita. La vibración del enlace $\mathrm{C}=\mathrm{N}$ la cual aparece a $1603 \mathrm{~cm}^{-1}$ en el ligando libre y a $1629 \mathrm{~cm}^{-1}$ cuando la 2-ampy esta coordinada, indica que estos enlaces se hacen mas largos y por lo tanto más débiles, los que es consistente con una retrodonación de densidad de carga desde los orbitales $d \pi$ llenos del metal.

Tabla 4. Espectro infrarrojo del complejo $\mathrm{RhCl}\left(\mathrm{PPh}_{3}\right)_{2}$ (2-ampy)

\begin{tabular}{cccc}
\hline Frecuencia $\left(\mathrm{cm}^{-1}\right)$ & asignación & Frecuencia $\left(\mathrm{cm}^{-1}\right)$ & asignación \\
\hline $3395 \mathrm{mf}$ & $v_{\text {as }} \mathrm{N}-\mathrm{H}$ & $1429 \mathrm{mf}$ & $\nu \mathrm{C}=\mathrm{C}\left(\mathrm{PPh}_{3}\right)$ \\
$3313 \mathrm{f}$ & $v_{\mathrm{s}} \mathrm{N}-\mathrm{H}$ & $1086 \mathrm{mf}$ & $\gamma \mathrm{C}-\mathrm{H}\left(\mathrm{PPh}_{3}\right)$ \\
$3051 \mathrm{f}$ & $v=\mathrm{C}-\mathrm{H}$ & $865 \mathrm{~m}$ & $\gamma \mathrm{C}-\mathrm{H}(2-\mathrm{ampy})$ \\
$1629 \mathrm{f}$ & $\nu \mathrm{C}=\mathrm{N}$ & $743 \mathrm{~m}$ & $\rho \mathrm{C}=\mathrm{C}-\mathrm{H}\left(\mathrm{PPh}_{3}\right)$ \\
$1560 \mathrm{~m}$ & $v \mathrm{C}=\mathrm{N}, \delta \mathrm{N}-\mathrm{H}$ & $702 \mathrm{mf}$ & $\rho \mathrm{C}=\mathrm{C}-\mathrm{H}\left(\mathrm{PPh}_{3}\right)$ \\
$1490 \mathrm{f}$ & $v \mathrm{C}=\mathrm{C}\left(\mathrm{PPh}_{3}\right)$ & $518 \mathrm{mf}$ & $\nu \mathrm{P}-\mathrm{C}$ \\
$1487 \mathrm{f}$ & $v \mathrm{C}=\mathrm{C}\left(\mathrm{PPh}_{3}\right)$ & & \\
\hline
\end{tabular}

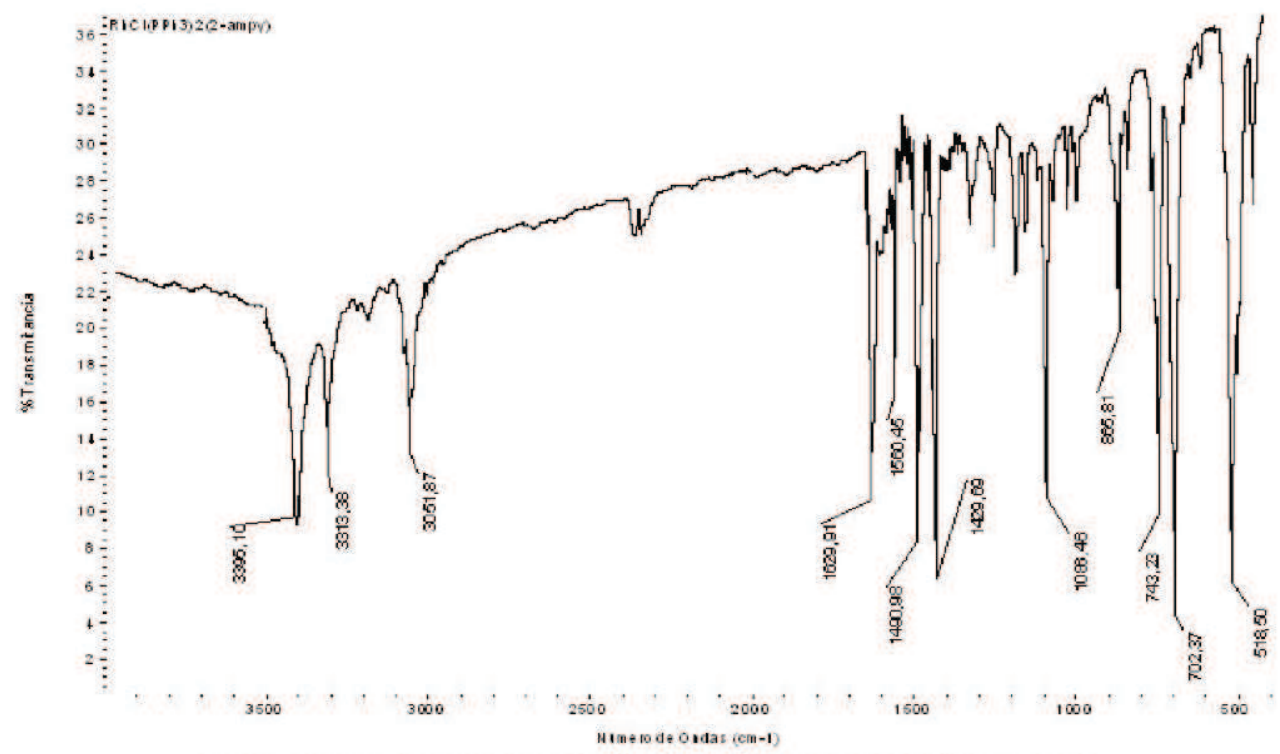

Figura 4. Espectro infrarrojo del complejo $\mathrm{RhCl}\left(\mathrm{PPh}_{3}\right)_{2}$ (2-ampy) 
La vibración simétrica del enlace $\mathrm{N}-\mathrm{H}$ que en el ligando libre se presenta a $3307 \mathrm{~cm}^{-1}$, cuando esta coordinado al metal aparece a $3313 \mathrm{~cm}^{-1}$, mostrando que es menos afectada que el modo vibracional asimétrico.

La vibración de estiramiento $\mathrm{C}-\mathrm{H}$ perteneciente a los anillos bencénicos de la trifenilfosfina libre se observa a $3067 \mathrm{~cm}^{-1}$, mientras que en el complejo se presenta a $3051 \mathrm{~cm}^{-1}$, mostrando así que cuando el metal realiza la retrodonación de densidad electrónica en el fósforo, aumenta la densidad electrónica en el fósforo fortaleciendo el enlace P-C, y debilitando los enlaces $\mathrm{C}-\mathrm{H}$, lo cual es confirmado en las regiones de bajas frecuencias en donde la frecuencia del enlace $\mathrm{P}$ $C$ del ligando libre aparece a $490 \mathrm{~cm}^{-1}$ y cuando esta coordinado al metal se presenta a $518 \mathrm{~cm}^{-1}$.
Los desdoblamientos por fuera del plano de los enlaces $\mathrm{C}=\mathrm{C}-\mathrm{H}$ de las trifenilfosfinas aparecen a $743 \mathrm{y}$ $702 \mathrm{~cm}^{-1}$. Estas frecuencias vibracionales muy intensas son características de los anillos aromáticos monosustituidos.

\section{RMN}

\section{$\mathrm{RMN}^{31} \mathrm{P}$}

El espectro del complejo presenta un doblete a 29.40 ppm con una constante de acoplamiento Rh-P de 96 $\mathrm{Hz}$, solvente $\mathrm{CDCl}_{3}$, figura 5 . Lo anterior es consistente con trifenilfosfinas en posición trans, por poseer el mismo ambiente químico.

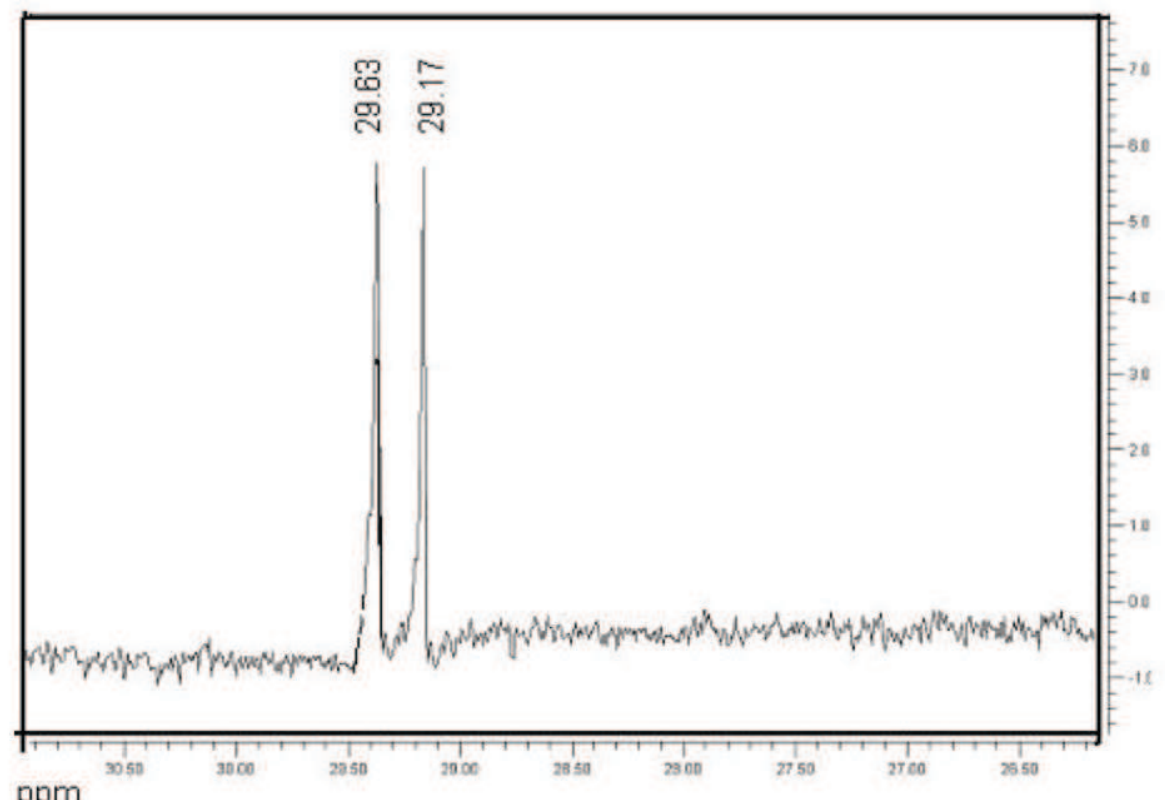

ppm

Figura 5. Espectro $\mathrm{rmn}^{31} \mathrm{P}$ del complejo $\mathrm{RhCl}\left(\mathrm{PPh}_{3}\right)_{2}$ (2-ampy)

\section{$\mathrm{RMN}^{1} \mathrm{H}$}

El espectro de rmn ${ }^{1} \mathrm{H}$ presenta seis señales (figura 6). La señal debida a los protones del grupo amino aparecen a $4.4 \mathrm{ppm}$ integrando para dos protones $y$ en el ligando libre a $4.6 \mathrm{ppm}$, corroborando que el metal realiza transferencias de densidad electrónica al anillo piridiníco a través del nitrógeno enlazado al metal aumentando la densidad de carga en las posiciones orto y para, lo cual genera que los protones del grupo amino se desplacen a campo alto. 


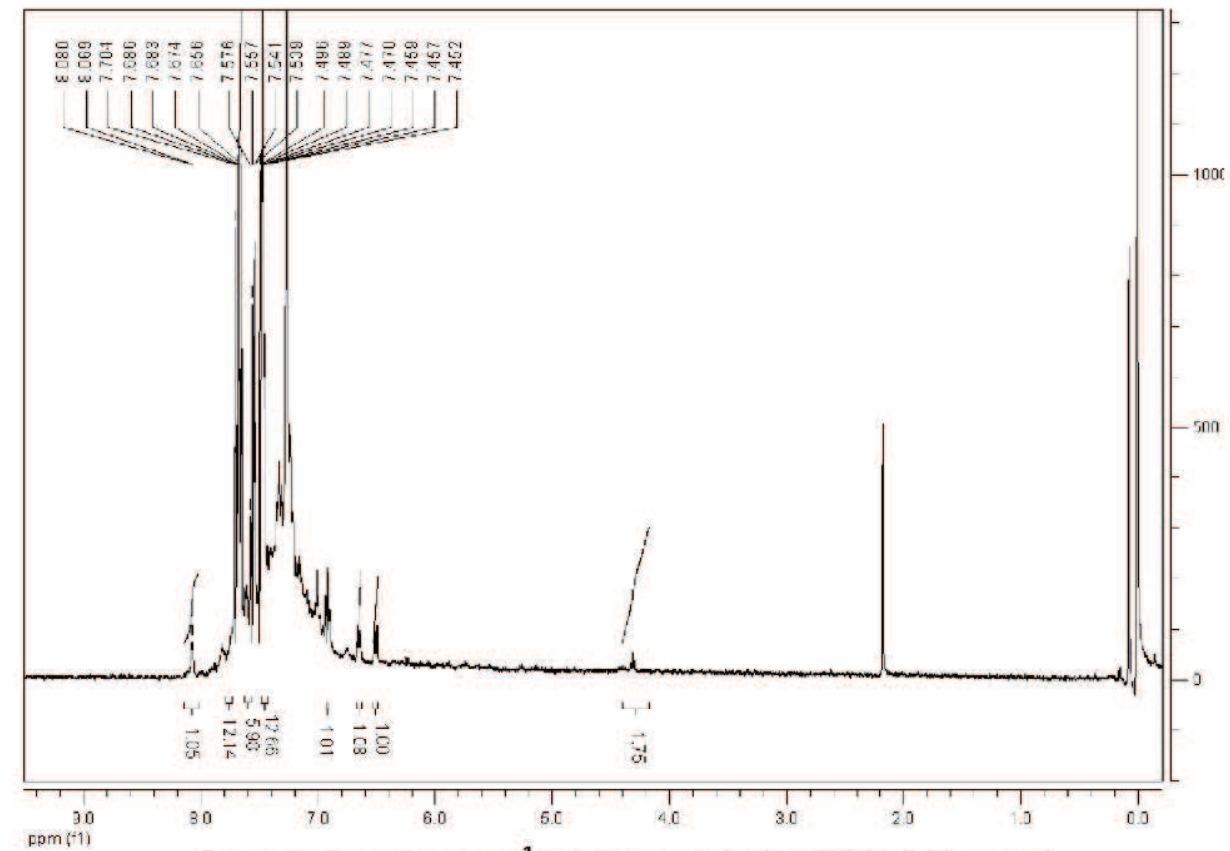

Figura 6. Espectro $\mathrm{rmn}^{1} \mathrm{H}$ del complejo $\mathrm{RhCl}\left(\mathrm{PPh}_{3}\right)_{2}(2$-ampy)

En la figura 6, se observa un doblete a $6.50 \mathrm{ppm}$ que integra para un protón el cual en el ligando libre se observa a $6.47 \mathrm{ppm}$, perteneciendo al protón $\mathrm{Hd}$ de la aminopiridina ver esquema 2 . El triplete observado a $6.64 \mathrm{ppm}$ que integra para un protón es asignado al hidrógeno $\mathrm{Hc}$ de la 2-ampy, en el ligando libre se observa a $6.60 \mathrm{ppm}$. El protón $\mathrm{Hb}$ se observa como un triplete que integra para un protón y presenta un desplazamiento a campo alto con respecto a la señal del ligando libre que se observa a $7.37 \mathrm{ppm}$. El protón $\mathrm{Ha}$ de la 2-ampy, se observa como un doblete a $8.073 \mathrm{ppm}$ que integra para un protón y en el ligando libre aparece a $8.04 \mathrm{ppm}$.

Los protones de las trifenilfosfinas se observan como un multiplete que integra para 30 protones entre 7.8 y $7.5 p p m$.

Los datos espectroscópicos indican que las trifenilfosfinas se encuentran en posición trans, que el ligando 2-aminopiridina se encuentra unido a través del nitrógeno piridiníco, por lo tanto la geometría del complejo es cuadrado planar y por lo tanto su grupo puntual es $C_{2 v}$, ver esquema 4 .

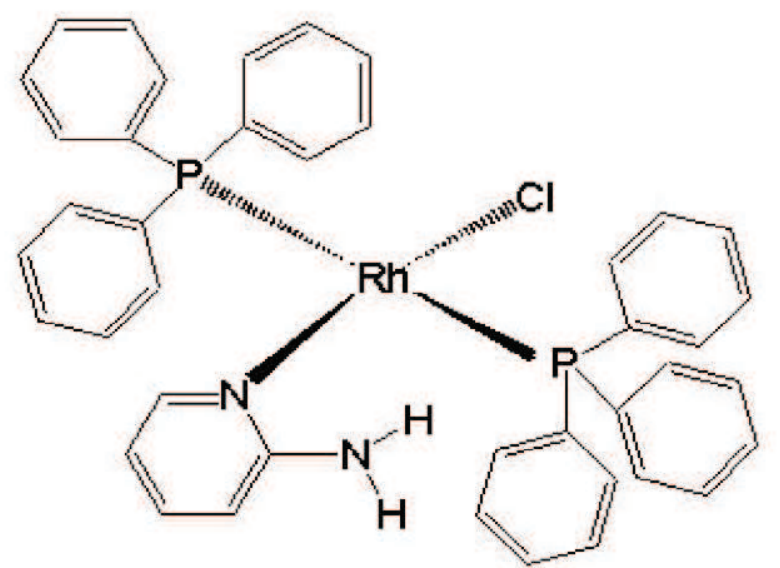

Esquema 4. Estructura del complejo $\mathrm{RhCl}\left(\mathrm{PPh}_{3}\right)_{2}$ (2-ampy)

\section{Hidroformilación de eugenol}

En la tabla 5, se muestra los resultados de la hidroformilación de eugenol con los complejos $\mathrm{RhCl}\left(\mathrm{PPh}_{3}\right)_{2}$ (2-ampy) y $\mathrm{RhCl}(1,5$-COD)(2-ampy), se observa que la selectividad y actividad catalítica de los complejos es muy diferente. El RhCl(1,5-COD)(2ampy), presenta la muy activo, llegando a un $100 \%$ de conversión de eugenol en 5 horas, mientras que $\mathrm{RhCl}\left(\mathrm{PPh}_{3}\right)_{2}(2$-ampy), en 5 horas de reacción solo lleva un $5 \%$ de conversión de eugenol, solo después de 24 horas llega al 100\% de conversión. 
Cuenú C., F., Duque H., M.C., Bolaños R., A. - 159 -

Tabla 5. Porcentajes de selectividad en la hidroformilación de eugenol con los complejos de rodio

\begin{tabular}{|c|c|c|c|c|c|}
\hline \multirow[b]{2}{*}{ Tiempo } & \multicolumn{2}{|c|}{$\begin{array}{c}\mathrm{RhCl}\left(\mathrm{PPh}_{3}\right)_{2} \\
(2-\mathrm{ampy})\end{array}$} & \multirow{2}{*}{$\begin{array}{c}\text { RhCl(COD) } \\
(2 \text {-ampy) } \\
5 \text { horas }\end{array}$} & \multirow{2}{*}{$\begin{array}{c}\mathrm{RhCl}\left(\mathrm{PPh}_{3}\right)_{3} \\
5 \text { horas }\end{array}$} & \multirow{2}{*}{$\begin{array}{c}{[\mathrm{RhCl}(\mathrm{COD})]} \\
5 \text { horas }\end{array}$} \\
\hline & 5 horas & 24 horas & & & \\
\hline Conv. Eugenol & 5.27 & 100.00 & 100.00 & 80.47 & 100.00 \\
\hline Isoeugenol & 6.02 & 4.26 & 42.10 & 12.01 & 55.60 \\
\hline Aldehído 1 & 0.00 & 11.20 & 0.00 & 6.16 & 5.07 \\
\hline Aldehído 2 & 23.71 & 34.97 & 20.60 & 25.22 & 17.89 \\
\hline Aldehído 3 & 59.40 & 43.65 & 37.30 & 53.46 & 21.44 \\
\hline areno & 10.87 & 2.03 & 0.00 & 3.15 & 0.00 \\
\hline Alcohol 1 & 0.00 & 1.68 & 0.00 & 0.00 & 0.00 \\
\hline Alcohol 2 & 0.00 & 1.46 & 0.00 & 0.00 & 0.00 \\
\hline Alcohol 3 & 0.00 & 0.75 & 0.00 & 0.00 & 0.00 \\
\hline
\end{tabular}

Condiciones: Temperatura 80 C, relación catalizador sustrato 1:3000, solvente benceno $20 \mathrm{~mL}$, presión 40 bar $\left(\mathrm{H}_{2} / \mathrm{CO}, 1: 1\right)$.

Con respecto a la selectividad se observa que $\mathrm{RhCl}(1,5-\mathrm{COD})(2$-ampy), presenta reacciones competitivas entre la hidroformilación y la isomerización del eugenol, pero no presenta productos de hidrogenación como los arenos o alcoholes. Además, no genera el aldehído A1 (aldehído $\alpha$, ver esquema 5). A pesar que en la hidroformilación de eugenol utilizando como precursor catalítico el complejo $\mathrm{RhCl}(1,5-\mathrm{COD})(2-$ ampy), hay una alta selectividad hacia el isoeugenol (42\%), no hay presencia del aldehído A1. Lo cual indica al complejo le es muy difícil interaccionar con el isoeugenol. 


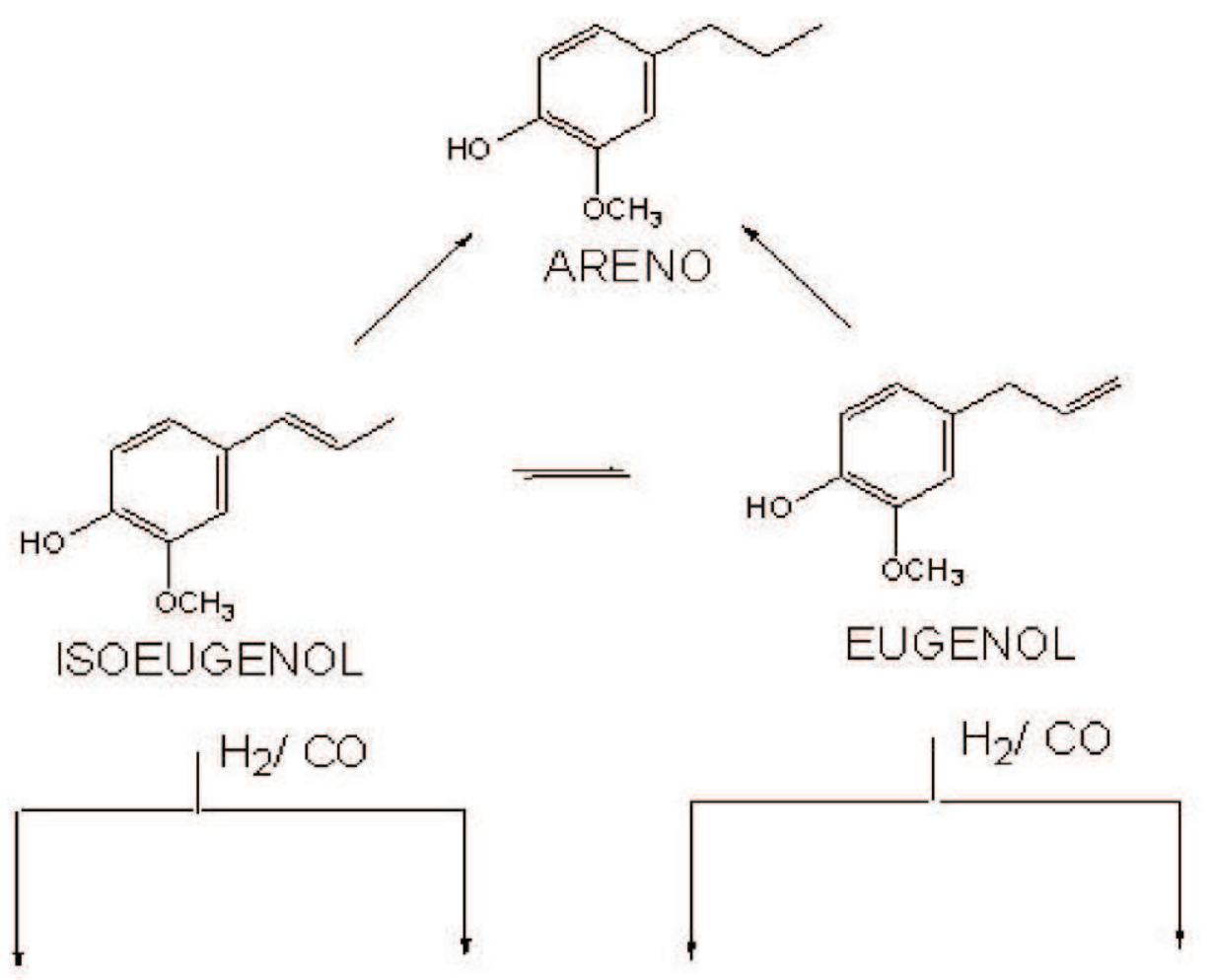<smiles>CCC(C=O)c1ccc(O)c(OC)c1</smiles>

Aldehído A1<smiles>CCC(CO)c1ccc(O)c(OC)c1</smiles>

Alcohol AL 1<smiles>COc1cc(CC(C)C=O)ccc1O</smiles>

Aldehido A2

$\mathrm{H}_{2}$<smiles>COc1cc(CC(C)CO)ccc1O</smiles>

A.lcohol A.L2<smiles>COc1cc(CCCC=O)ccc1O</smiles>

Aldehído A3<smiles>COc1cc(CCCCO)ccc1O</smiles>

Alcohol AL3

Esquema 5. Productos de la hidroformilación de eugenol 
El complejo $\mathrm{RhCl}(1,5-\mathrm{COD})$ (2-ampy), presenta la misma actividad que el precursor $[\mathrm{RhCl}(1,5-\mathrm{COD})]_{2}$, pero el complejo con la 2-ampy, presenta una mayor selectividad hacia el aldehído lineal (A3), mientras que el diméro tiene una selectividad hacia el aldehído A1, mostrando que no es selectivo ya que ataca tanto al eugenol como al isoeugenol, mientras que el complejo con la 2-ampy, no es selectivo al aldehído $\mathrm{A} 1$, lo que sugiere que no ataca al isoeugenol.

El complejo $\mathrm{RhCl}\left(\mathrm{PPh}_{3}\right)_{2}$ (2-ampy), en las primeras horas de reacción es poco quimioselectivo, debido a que presenta una alta selectividad hacia el areno, el cual es producto de la hidrogenación del isoeugenol (10\%), además presenta un $6 \%$ de selectividad hacia la isomerización de eugenol. Con respecto a los productos oxo, el catalizador es más selectivo hacia el aldehído lineal. Ahora cuando el catalizador llega a un $100 \%$ de conversión, la selectividad hacia los productos oxo es de aproximadamente el $90 \%$, y la selectividad hacia la hidrogenación del eugenol y la isomerización disminuyen a un 2 y 4 \% respectivamente. Además se observan los productos de la hidrogenación de los aldehídos con porcentajes de selectividad que no alcanzan el $3 \%$.

Tabla 6. Efecto de la temperatura en la hidroformilación de eugenol con el complejo $\left.\mathrm{RhCl}_{(\mathrm{PPh}}\right)_{2}(2-\mathrm{ampy})$

\begin{tabular}{ccccc}
\hline To (tiempo) & $60 \circ \mathrm{C}(48 \mathrm{~h})$ & $80 \circ \mathrm{C}(24 \mathrm{~h})$ & $100 \circ \mathrm{C}(5 \mathrm{~h})$ & $100 \circ \mathrm{C}(10 \mathrm{~h})$ \\
\hline Conv. Eugenol & 100.00 & 100.00 & 100.00 & 100.00 \\
Isoeugenol & 3.24 & 4.26 & 82.50 & 42.39 \\
Aldehído 1 & 0.00 & 11.20 & 0.00 & 9.70 \\
Aldehído 2 & 19.81 & 34.97 & 8.42 & 20.94 \\
Aldehído 3 & 75.25 & 43.65 & 9.08 & 19.39 \\
areno & 1.70 & 2.03 & 0.00 & 5.20 \\
Alcohol 1 & 0.00 & 1.68 & 0.00 & 2.38 \\
Alcohol 2 & 0.00 & 1.46 & 0.00 & 0.00 \\
Alcohol 3 & 0.00 & 0.75 & 0.00 & 0.00 \\
\hline
\end{tabular}

Condiciones: relación catalizador sustrato 1:3000, solvente benceno $20 \mathrm{~mL}$, presión 40 bar $\left(\mathrm{H}_{2} / \mathrm{CO}, 1: 1\right)$.

Puesto que, el complejo $\mathrm{RhCl}\left(\mathrm{PPh}_{3}\right)_{2}$ (2-ampy), presenta mayores selectividades hacia el aldehído lineal, se realizó el estudio del efecto de la temperatura en la hidroformilación de eugenol, los datos se presentan en la tabla 6 , en donde se determina que la temperatura afecta tanto a la actividad como a la selectividad, así a 60 으, el complejo es regioselectivo hacia los productos oxo, además la selectividad hacia el aldehído lineal es del $75 \%$. Al aumentar la temperatura de reacción el complejo aumenta la selectividad hacia la isomerización del eugenol y la hidrogenación del eugenol y del aldehído A1.

El precursor $\mathrm{RhCl}\left(\mathrm{PPh}_{3}\right)_{3}$, presenta una mayor actividad que el complejo $\mathrm{RhCl}\left(\mathrm{PPh}_{3}\right)_{2}$ (2-ampy), además en un $100 \%$ de conversión es más selectivo hacia el aldehído lineal A3 y una menor selectividad hacia el aldehído $A 2$, siendo consistente con una especie activa con altos impedimentos estéricos y una mayor cantidad de ligantes fosfinas, los cuales inducen un mayor impedimento estérico y además son excelentes donores de densidad de carga, lo que favorece las reacciones de adición oxidativa de hidrógeno. Así se puede indicar que el complejo con el ligante 2-ampy, presenta una menor actividad catalítica debido a que la 2-ampy es un ligante que induce menores impedimentos estéricos, lo que se refleja en una alta selectividad del aldehído $A 2$ y del aldehído A1.

\section{Hidroformilación de isoeugenol}

La tabla 7, muestra que en la hidroformilación de isoeugenol la actividad de los complejos es baja, y el complejo $\mathrm{RhCl}(1,5-\mathrm{COD})(2$-ampy) presenta una mayor actividad y es más selectivo hacia el aldehído A1. A pesar que no se observa la presencia del eugenol, hay producción del isoeugenol, lo que indica, que la velocidad de conversión del eugenol es mayor a la del isoeugenol.

El complejo $\mathrm{RhCl}\left(\mathrm{PPh}_{3}\right)_{2}$ (2-ampy), presenta un mayor actividad hacia el aldehído A2, y la selectividad hacia el aldehído lineal (A3), es mayor que en el compuesto $\mathrm{RhCl}(\mathrm{COD})(2$-ampy), lo anterior es consistente con una mayor isomerización del isoeugenol en el complejo con fosfinas, lo que aumenta la selectividad hacia el aldehído lineal y por lo tanto disminuye la selectividad hacia el aldehído A1. 
Tabla 7. Porcentajes de selectividad en la hidroformilación de isoeugenol con los complejos de rodio

\begin{tabular}{|c|c|c|c|c|}
\hline & \multicolumn{2}{|c|}{$\mathrm{RhCl}\left(\mathrm{PPh}_{3}\right)_{2}$ (2-ampy) } & \multicolumn{2}{|c|}{$\mathrm{RhCl}(1,5-\mathrm{COD})(2-\mathrm{ampy})$} \\
\hline & 5 horas & 24 horas & 5 horas & 24 horas \\
\hline Eugenol & 0.00 & 0.00 & 0.00 & 0.00 \\
\hline Conv. Isoeugenol & 26.75 & 78.73 & 46.60 & 100 \\
\hline Aldehído 1 & 36.89 & 37.34 & 49.31 & 52.36 \\
\hline Aldehído 2 & 44.89 & 45.55 & 41.15 & 43.25 \\
\hline Aldehído 3 & 18.22 & 13.66 & 9.54 & 3.34 \\
\hline areno & 0.00 & 3.45 & 0.00 & 1.05 \\
\hline
\end{tabular}

Los estudios catalíticos de las reacciones de hidroformilación de eugenol e isoeugenol con los compuestos aquí reportados, indican que el ligante 2 ampy, se hace presente en las especies cataliticamente activas de los complejos y que, la especie catalítica en la reacción con el complejo $\mathrm{RhCl}\left(\mathrm{PPh}_{3}\right)_{2}$ (2-ampy), es diferente a la del compuesto $\mathrm{RhCl}(\mathrm{COD})(2$-ampy), en donde el complejo con el ligando 1,5-COD, fácilmente reemplaza este ligando por grupos carbonilos, así la especie cataliticamente tendrá los ligandos carbonilos y la 2-ampy. Mientras que en el complejo $\mathrm{RhCl}\left(\mathrm{PPh}_{3}\right)_{2}$ (2-ampy), la 2-ampy, que posse un enlace mas fuerte que la fosfina, estará en la especie activa así se considera que la activación de los dos compuestos sería la observada en la figura 7. En donde la activación de la molécula se da a través de una activación heterolítica catalizada por un base, que en este caso es la 2-ampy coordina. Además se da la coordinación al metal de ligantes carbonilos, los cuales se coordinan muy fácil a metales de transición con bajos estados de oxidación y orbitales $d \pi$ llenos.

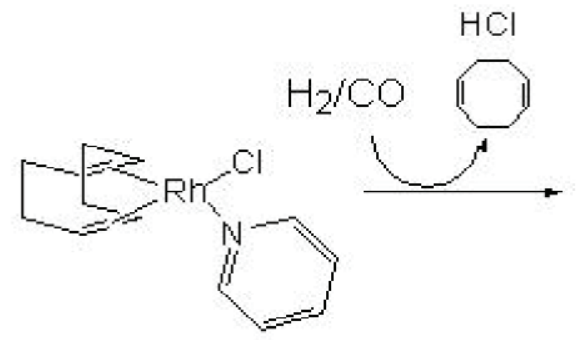<smiles></smiles>

[Rh 1]<smiles></smiles>

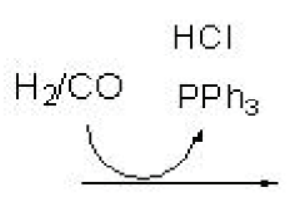<smiles></smiles>

[Rh 2]

Figura 7. Posible generación de las especies catalíticas. 
En la figura 8, se propone un mecanismo de reacción para la hidroformilación de eugenol con el complejo $\mathrm{RhCl}\left(\mathrm{PPh}_{3}\right)_{2}$ (2-ampy), en donde la especie activa inicialmente coordina al eugenol a través de un enlace $\pi$ (paso 1), generando una especie intermedia pentacoordinada de 18 electrones, la cual rápidamente sufre la migración del hidruro, que puede atacar al carbono terminal o al carbono $\beta$, ahora si hay altos impedimentos estéricos en los ligantes, se formará el intermediario rodio-alquilo (lineal) de 18 electrones, con una geometría cuadrado planar (paso 2). Éste intermediario rodioalquilo, puede sufrir tres reacciones, la primera es la inserción del grupo carbonilo, entre el grupo alquilo y el rodio, con la formación del intermediario rodioacilo (paso3), que luego sufre una adición oxidativa de hidrógeno y una posterior eliminación reductiva para producir el aldehído $\mathrm{A} 3$ y regenerándose la especie cataliticamente activa [Rh 2] (paso 4).

Ahora, si el intermediario Rh-alquilo, sufre una adición oxidativa de hidrógeno (paso 7), se formará un intermediario de $\mathrm{Rh}(\mathrm{III})$, de 18 electrones y por su alto estado de oxidación y una alta saturación coordinativa, rápidamente realiza una eliminación reductiva con la formación del areno y la regeneración de la especie cataliticamente activa.

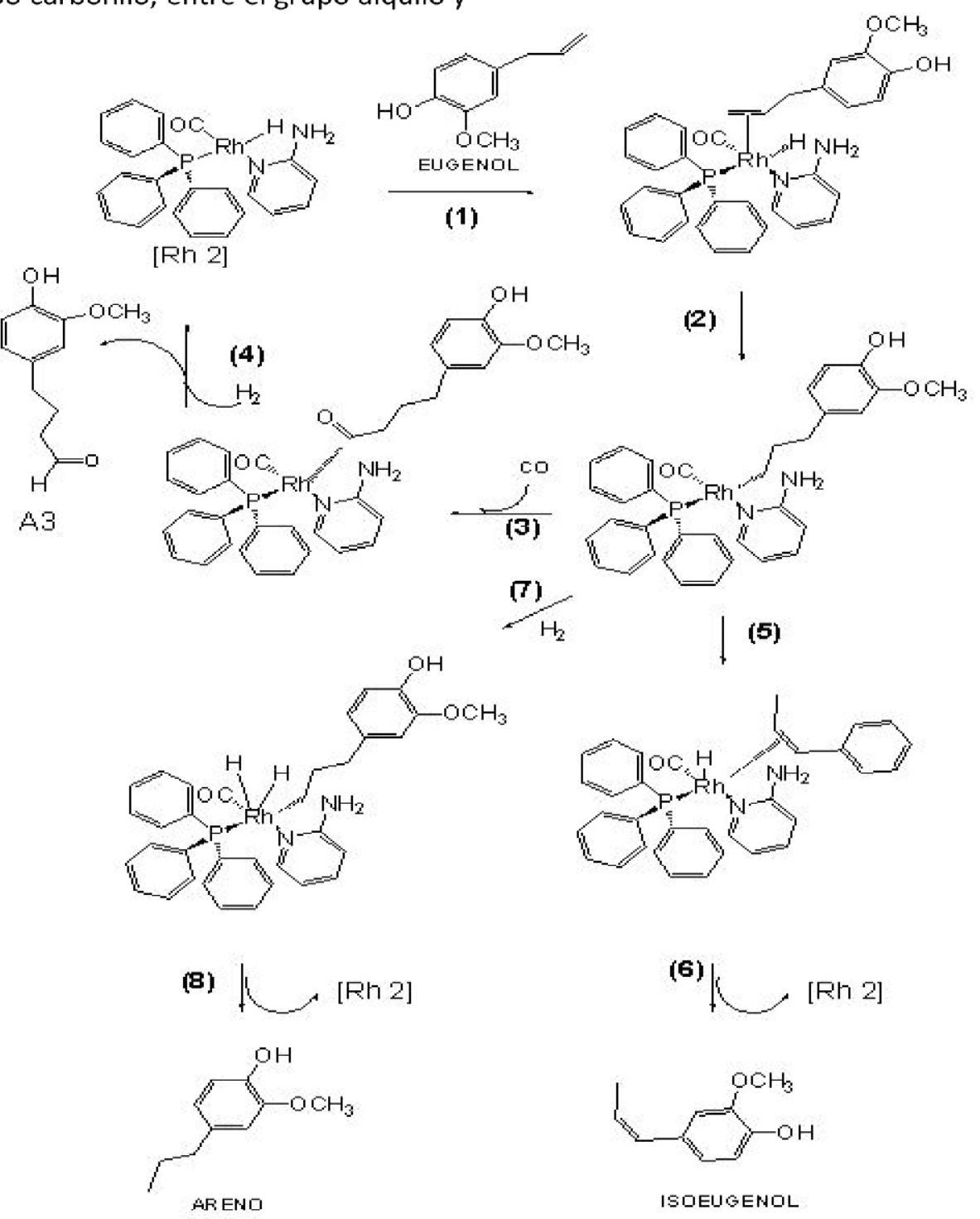

Figura 8. Mecanismo propuesto para la hidroformilación de eugenol con la especie [Rh 2]. 
La tercera reacción que sufre la especie rodio-alquilo es la reacción de isomerización, en donde ésta especie rodio-alquilo, sufre una eliminación de hidrógeno $\beta$ (paso 5), con la formación del isoeugenol y la regeneración de la especie activa.

\section{CONCLUSIONES}

Los espectros rmn indican que el complejo $\mathrm{RhCl}\left(\mathrm{PPh}_{3}\right)_{2}$ (2-ampy), posee las trifenilfosfinas en una configuración trans, debido a que presentan un doblete a $29.4 \mathrm{ppm}$ con una constantes de acoplamiento de $96 \mathrm{~Hz}$, así la geometría del compuesto es cuadrado planaren el espectro de rmn ${ }^{31} \mathrm{P}$. El complejo es activo hacia las reacciones de hidroformilación de eugenol e isoeugenol. Los complejos $\mathrm{RhCl}\left(\mathrm{PPh}_{3}\right)_{2}$ (2-ampy) y $\left[\mathrm{RhCl}\left(\eta^{4}-1,5-\right.\right.$ COD)(2-ampy)], presentan reacciones competitivas de hidroformilación y de isomerización, además en un menor porcentaje reacciones de isomerización, debido a que el ligante 2-ampy, genera bajos impedimentos estéricos, pero no es un buen donor de densidad de carga, lo que favorece que no realice fácilmente reacciones de hidrogenación, en especial el complejo con el ligante COD.
Los estudios del efecto de la temperatura indican que el complejo $\mathrm{RhCl}\left(\mathrm{PPh}_{3}\right)_{2}$ (2-ampy) a bajas temperaturas es muy poco activo, pero es muy selectivo hacia el aldehído lineal y al aumentar la temperatura se hace más selectivo hacia las reacciones de isomerización, lo que genera un aumento en la selectividad de los aldehídos ramificados.

En la hidroformilación de isoeugenol, los complejos se comportan de manera diferente, el complejo con los grupos fosfina es menos activo y presenta reacciones competitivas de entre la isomerización y la hidroformilación, lo que produce una mayor selectividad hacia el aldehído $\beta$ (A2), mientras que el compuesto con el grupo carbonilo es más activo y más selectivo hacia el aldehído $\alpha$ (A1).

\section{AGRADECIMIENTOS}

Se agradece a la Universidad del Valle, Colciencias y a la Universidad del Quindío por la financiación de este proyecto.

\section{BIBLIOGRAFÍA}

\section{A. J. Pardey, C. Longo, Coord. Chem. Rev. 254 (2010) 254}

2. A. Togni, L.M. Venanzi, Angew. Chem. Int. Ed. Engl. 33 (1994) 497; (b) J.D. Petersen, W.R. Murphy, R. Sahai, K.J. Brewer, R.R. Rumenski, Coord. Chem. Rev. 261 (1985) 64; (c) R.G. Pearson, Science 151 (1966) 172.; (d) G. Erre, S. Enthaler, K. Jungle, J. Mol. Cat: A. Chemical, 280 (2008) 148.; (e) M. Dièguez, O. Pàmies, C. Claver, Tetrahedron: assymmetry, 15 (2004) 2113. (f) S. Gladiali, J. Bayón, C. Claver, Tetrahedron: assymmetry, 6, (1995), 1453. (g) B. Bantu, K. Wurst, M. Buchmeiser, J. Organomet. Chem. 692 (2007) 5272.

3. P. Pino, G. Consiglio, C. Botteghi, C. Salomón, Adv. Chem. Ser. 132 (1974) 295.

4. C. Botteghi, S. Paganelli, A. Schionato, M. Marchetti, Chirality 3 (1999) 355.

5. K. Bauer, D. Garbe, H. Surburg, Common Fragance and Flavors Materials: Preparation and Uses, Wiley-VCH, Germany, 2001

6. C.M. López, D. Arias, F.J. Machado y A. Tuek. Cat. Lett. 74 (2001) 163

7. E. Ponzi, O. Masini, N. Cornelli, L. Grzona, A. Carrusoull y M. Ponzi, Bol. Soc. Chil. 44 (1999) 271.

8. K.A.D. Swift, Topics in Catal. 27 (2004) 143. 
9. C. Choi, K. Park, J. Lee, Y. Jeon, K. Liu, S. Oh, D. Kim, S. Yea, Eur. J. Pharm. 576 (2007) 151.; (b) W.E. Erman, Chemistry of the Monoterpenes. An Encyclopedic Handbook, Marcel Dekker, New York, 1985.; (c) K.A. Da Silva, I.V. Kozhevnikov, E.V. Gusevskaya, J. Mol. Catal. A 192 (2003) 129.

10. S. B. Owens, G. M. Gray, Organometallics, 27 (2008) 4282.; (b)nKlosin, J.; Landis, C. R. Acc. Chem. Res. 2007, 40, 1251.;(c) Babin, J. E.; Whiteker, G. T. WO 9303839, 1993.; (d) Dieguez, M.; Pamies, O.; Ruiz, A.; Castillon, S.; Claver, C. Chem.Commun. (2000), 1607.; (e) Dieguez, M.; Pamies, O.; Ruiz, A.; Castillon, S.; Claver, C. Chem. Eur. J. 7 (2001) 3086.; (f) Dieguez, M.; Pamies, O.; Ruiz, A.; Claver, C. New J. Chem. 26 (2002) 827.; (g) K. A. Chatziapostolou, K. A. Vallianatou, A. Grigoropoulos,

C. P. Raptopoulou, A. Terzis, I. D. Kostas,

P. Kyritsis, G. Pneumatikakis, Journal of Organometallic Chemistry 692 (2007) 4129.; (h) A. Moores, N. Mezailles, L. Ricard, P. F. Moores, Organometallics 24 (2005) 508.

11. A.C. da Silva, K.C.B. de Oliveira, E.V. Gusevskaya, E.N. dos Santos, J. Mol. Catal. A: Chem. 179 (2002) 133.; (b) M. Rosa Axet, S. Castillón, Carmen Claver, Inorg. Chim. Acta 359 (2006) 2973.

12. L. Kollhar, E. Farkas, J. Batiu, J. Mol. Cat. A: Chemical 115 (1997) 283-288. ; (b) C. Botteghi, S. Paganelli, A. Schionato, M. Marchetti, Chirality 3 (1991) 355.; (c) B.M. Trost, I. Fleming (Eds.), Comprehensive Organic Synthesis, Vol. 2, Pergamon Press Ltd., 1991, pp. 754-763.; (d) D.H. Grayson, Nat. Prod. Rep. 5 (1988) 419.

13. E. Slivinskii,N. V. Kolesnichenko, Russ. Chem. Bull, Inter. Ed. 53 (2004) 2449; (b) A. J. Pardey, F. Hung-Lowa, G. C. Uzcáteguia, M. C. Ortega, C. Longo, React. Kinet. Catal. Lett. 88 (2006) 203.; (c) H. Guoa, S. Ma, Adv. Synth. Catal. 350, (2008), 1213.

14. V. Landaeta, M. Peruzzini, V. Herrera, C. Bianchini, R.

Sánchez-Delgado, A. Goeta, F. Zanobini, J. Organomet. Chem, 691 (2006) 1039. ; (b) P. Ribeiro, C. Donnici, E. N. dos Santos, J. Organomet. Chem, 691 (2006) 2037; (c) J. Rajput, A. Hutton, R. Moss, C. Imrie, J. Organomet. Chem, 691 (2006) 4573. ; (d) C. S. Vasama, S. Modema, S. Kankala,G. Budigea, R. Vadde, Appl. Organometal. Chem. 23 (2009) 460.

15. M. A. Garralda, R. Hernández, L. Ibarlucea, E. Pinilla, M. R. Torres, M. Zarandona, Organometallics 26 (2007) 5369.

16. M. A. Garralda, R. Hernández, L. Ibarlucea, M. I. Arrioutua, K. Urtiaga, Inorg. Chim. Acta, 232 (1995) 9.; (b) M. Beller, H. Trauthwein, M. Eichberger, C. Breindl, T. E. Müller, Alexander Zapf, J. Organomet. Chem 566 (1998) 277; (c) H. Brunner, B. Nuber, M. Prommesberger, J. Organomet. Chem., 523 (1996) 179.; (d) B. Bantu, K. Wurst, M. Buchmeiser, J. Organomet. Chem, 692 (2007) 5272. 